\title{
SIDESCAN SONAR IMAGE PROCESSING:CORRECTING BRIGHTNESS VARIATION AND PATCHING GAPS
}

\author{
Yet-Chung Chang \\ ycc@tsu.edu.tw \\ Shu-Kun Hsu \\ Department of Earth Sciences, National Central University, Taiwan, R.O.C \\ Ching-Hui Tsai \\ Department of Earth Sciences, National Central University, Taiwan, R.O.C
}

Department of Digital Entertainment and Game Design, Taiwan Shoufu University, Tainan County, Taiwan, R.O.C,

Follow this and additional works at: https://jmstt.ntou.edu.tw/journal

Part of the Electrical and Computer Engineering Commons

\section{Recommended Citation}

Chang, Yet-Chung; Hsu, Shu-Kun; and Tsai, Ching-Hui (2010) "SIDESCAN SONAR IMAGE PROCESSING:CORRECTING BRIGHTNESS VARIATION AND PATCHING GAPS," Journal of Marine Science and Technology. Vol. 18: Iss. 6, Article 1. DOI: $10.51400 / 2709-6998.1935$

Available at: https://jmstt.ntou.edu.tw/journal/vol18/iss6/1

This Research Article is brought to you for free and open access by Journal of Marine Science and Technology. It has been accepted for inclusion in Journal of Marine Science and Technology by an authorized editor of Journal of Marine Science and Technology. 


\title{
SIDESCAN SONAR IMAGE PROCESSING: CORRECTING BRIGHTNESS VARIATION AND PATCHING GAPS
}

\author{
Yet-Chung Chang*, Shu-Kun Hsu**, and Ching-Hui Tsai**
}

Key words: sidescan, sonar, brightness, gap patching, mosaic, footprint.

\section{ABSTRACT}

Sidescan sonar data are typically presented as gray level images. However, sidescan images often show striking variations in brightness. These variations, caused by the sonar beam pattern and the constantly changing attitude of the towfish, make the images difficult to read as pictures of the seabed. This reduces the utility of the images for marine geologists. In this paper, the effects of the sonar beam pattern across the swath are corrected using a normalization process based on the average signal intensity for each grazing angle. Noise along the track, which is generally caused by changes in the attitude of the towfish, is eliminated using an assumption that the total back-scattered energy from each ping should be similar to adjacent pings in the time series.

These two processes allow the production of smooth and clear waterfall displays. However, when the sidescan data are projected onto a map as a high resolution "mosaic" image, gaps appear on the outer edge of the swath where the towfish changes course. To remove the gaps, a patching algorithm is proposed. The patching method is justified theoretically by the shape of the sonar footprint on the sea floor. All the pixels falling inside the sonar footprint can be assigned reasonable values using just the original data series, with no need for interpolation.

\section{BASIC PROCESSING}

The sidescan sonar is a powerful, versatile but low cost tool for surveying the sea floor [1-5, 7]. Usually a ship tows a towfish mounted with two sonar arrays, one on each side. The sonar arrays emit fan-shaped sonar signals perpendicular to the direction of travel. The signals scan a swath of sea floor

Paper submitted 05/22/09; revised 12/14/09; accepted 01/08/10. Author for correspondence: Yet-Chung Chang (e-mail: ycc@tsu.edu.tw).

*Department of Digital Entertainment and Game Design, Taiwan Shoufu University, Tainan County, Taiwan, R.O.C.

**Department of Earth Sciences, National Central University, Taiwan, R.O.C. from a point just below the towfish to a limited distance away from the line of travel on both sides. The raw sonar data are two time series of digitized sound: the back-scattered signals from each ping on the port and starboard sides of the towfish. If we display the data as gray level pixels representing the acoustic energy, the result is a image similar to the left side of Fig. 1. This is called a waterfall display.

Images based directly on the raw time series data have a blind zone in the center, so for most purposes, we do not use the data in this raw form. The raw waterfall needs to be processed into an image which roughly corresponds to a plane area of the sea floor. The most basic form of processing for sidescan data is "slant range correction" $[5,6,8]$. For this process, we assume that the seabed is a perfect flat plane. Then, given the altitude of the towfish, and the time at which the backscatter reaches the sonar array, we can calculate a position on the seabed. After processing the data, the blind zone in the center of image disappears, and every datum is relocated to a position more representative of the actual seabed. Figure 1 shows an example of a waterfall image before and after slant range correction.
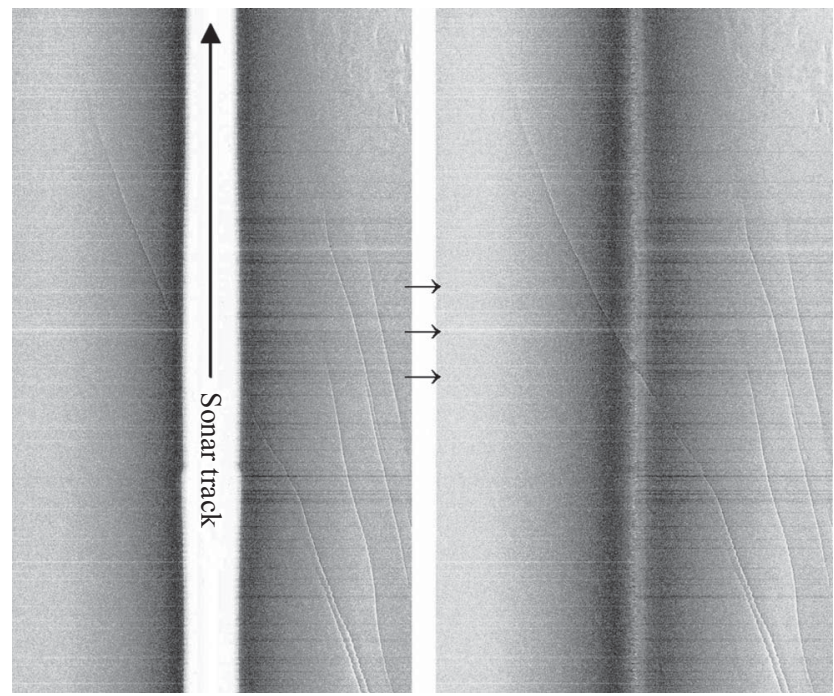

Fig. 1. Sidescan data before and after slant range correction. The scan range for each side is about $400 \mathrm{~m}$. The terrain features shown are several elongated small normal faults on a slope of sea bottom. 


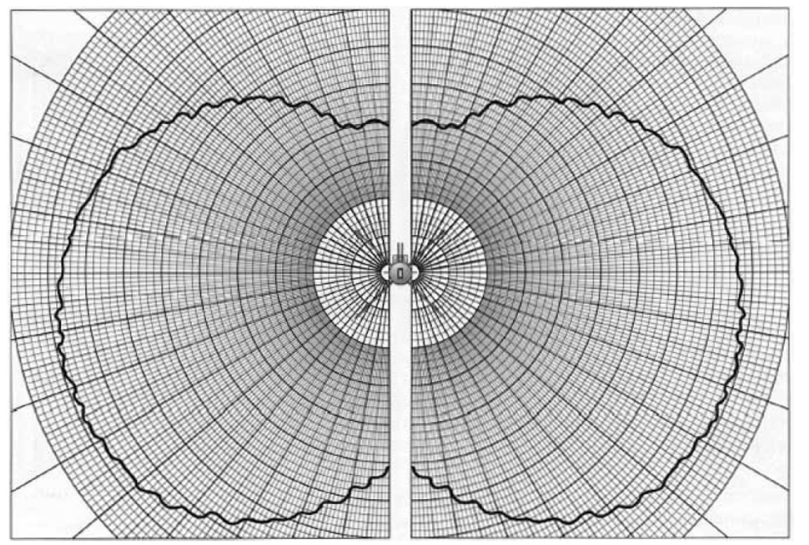

Fig. 2. A typical beam pattern for sidescan sonar equipment [6]. The solid curves represent the relative intensity of sonar emission in different directions.
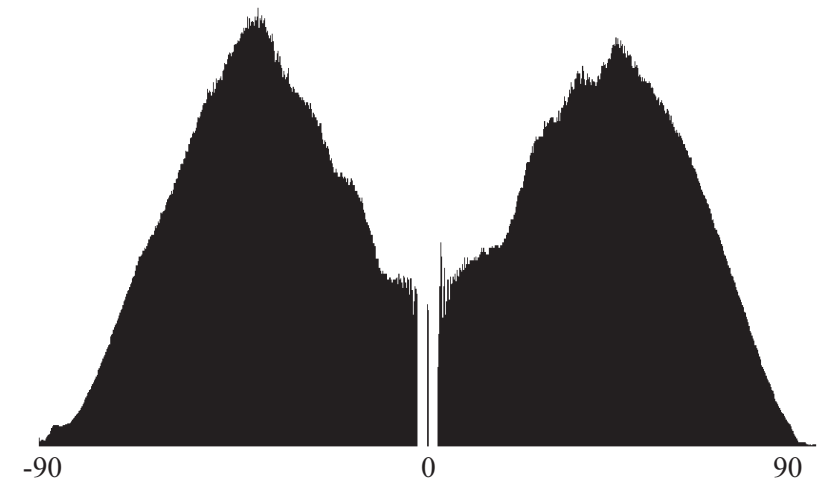

Fig. 3. Back-scattered energy as a function of grazing angle. The horizontal axis denotes the grazing angle; the vertical axis denotes the relative intensity.

All the images in this paper were processed based on 16-bit raw intensity data, where the darker gray color means higher intensity of backscattering. In general, the 16-bit raw data allows more flexible contrast exaggeration processing than the 8-bit data set. Most images in this paper had been enhanced in gray level contrast to some extent without any side effect of color discontinuity. If the case was 8-bit, many color discontinuity or bad quality within the images would be inevitable.

\section{BEAM PATTERN CORRECTION}

The most prominent feature of sidescan images like Fig. 1 is the too strong intensity near the path of the towfish, and the weak response at the outer edge of the swath. This is caused by the beam pattern of the sonar [8]. A typical sidescan sonar beam pattern is shown in Fig. 2. Across the swath, the amount of sonar energy hitting the seabed will vary with the distance from the towfish. The angle at which the sonar wave hits the sea floor (grazing angle) also varies. The raw sidescan sonar data are the back-scattered energy from the seabed; both the level of incident energy and grazing angle will affect the data.
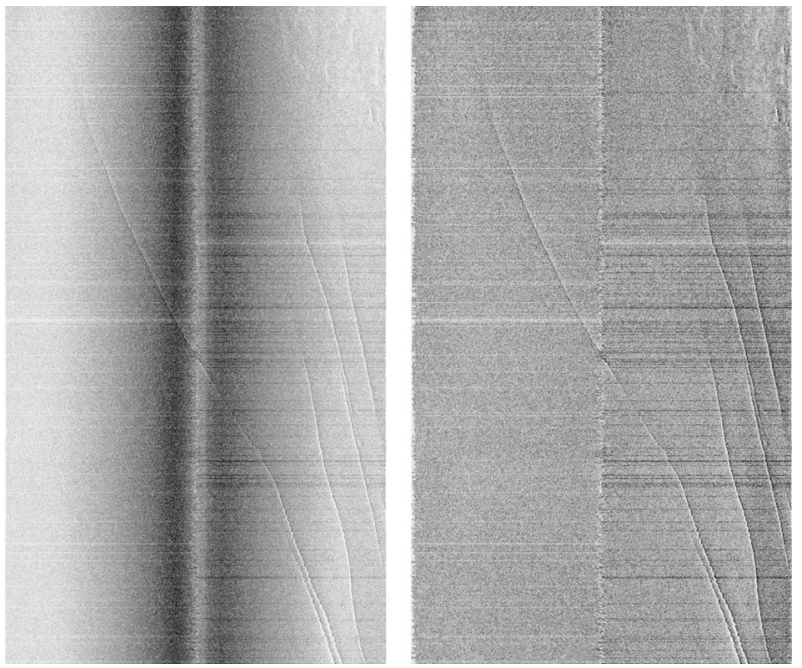

Fig. 4. Images before and after beam pattern correction.

Since the sidescan is actually two-sided sonar, each side has its own main lobe pointed outward from the vertical line about 45 degrees, the maximum intensity therefore located around this angle, rather than right below the towfish (see Fig. 2).

To compensate for the uneven illumination, we need to know the energy distribution function relative to the angle. One simple way to find the energy distribution is to sum up the energy levels for each angle over the whole data series. The result is a histogram like Fig. 3. Using this statistical result, we can calculate the average energy for each angle; the inverse of this average can be applied as a correcting factor to individual data in the time series. The images before and after this correction are shown in Fig. 4. The unwanted variation across the swath has been successfully removed.

Most processing procedures for the same image correction purpose are executed by time variable gains (TVG), either at source or in post-processing. However, the time based functions are not suitable to describe the variation of backscattering energy which is basically controlled by the grazing angle. For example, the TVG's must be continuously adjusted to adapt the depth changes. In the other hand, the proposed method based on grazing angle in this paper would be nearly invariant with the depth. It seems to be a more convenient and theoretically proper method for most data processors.

Since the function of beam pattern here was established by summing up the data set itself, it was possible to rule out some real signals right at certain grazing angle by this method. However, the possibility of certain signals always appear around certain grazing angle is rare. In most cases, the method has provided good performance.

\section{PING ENERGY LEVEL NORMALIZATION}

The energy broadcast by the sonar array should be exactly the same for each ping. However, as the towfish is pulled under the water, it is constantly moving because of waves and 


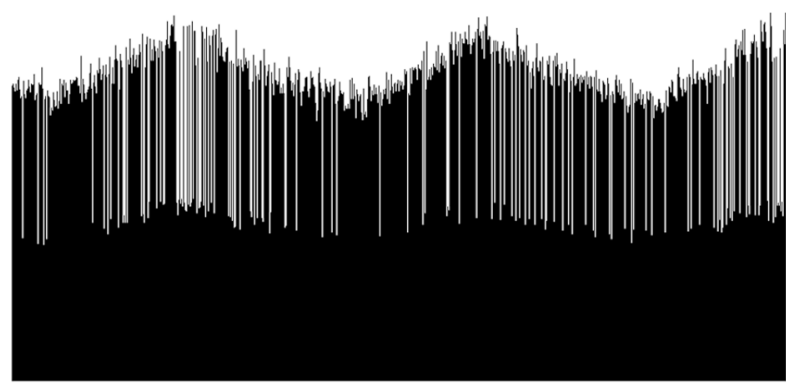

Fig. 5. Total ping energy levels. The horizontal axis denotes the series pings and the vertical axis denotes the average intensity of each ping.
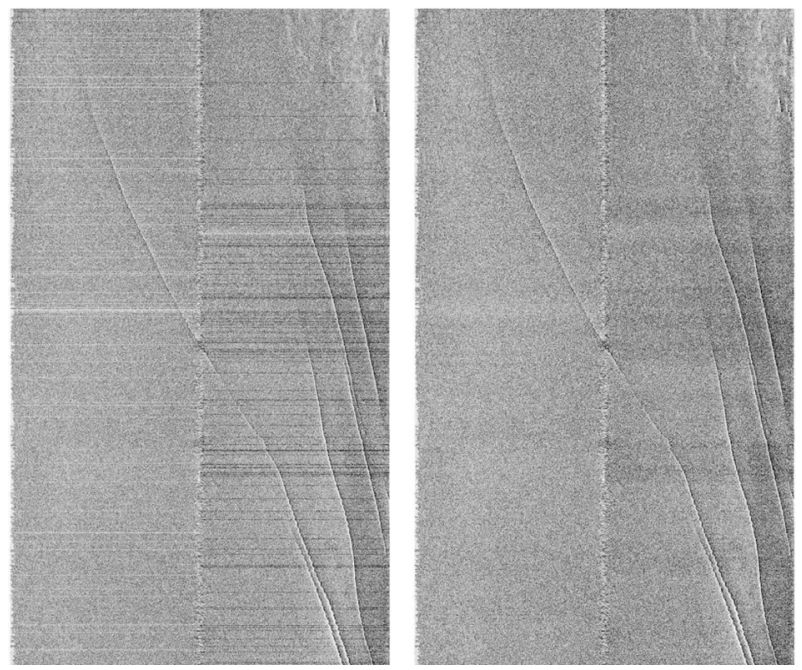

Fig. 6. Images before and after the ping energy level normalization.

currents. This causes the energy actually incident on the sea floor to vary from ping to ping. For example, rolling motion might cause the energy level to rise on one side and drop on the other side. The level of back-scattered energy also changes with the surface character of the seabed. So the energy levels can change abruptly between pings, and these abrupt changes show up as the white and black horizontal lines in Fig. 4.

To suppress this kind of noise, we assume that each ping "should" have a similar energy level to the pings before and after it. The total back-scattered energy level for each ping is calculated, and represented as the histogram in Fig. 5. For each ping, we find the average energy level of the 20 pings before and after it. This average is used as a reference value, and the total energy of the ping is smoothed to match it. The result is that the abrupt signal changes are mostly removed. The images before and after this process are shown in Fig. 6.

It should be mentioned that the assumption here does not always hold. The ping energy level does vary in reality when the character of the sea floor changes. That is why the smoothing must be done with reference to a fairly short sequence of pings (40 in this example). If the smoothing is too extensive, real changes in the sea floor will be obscured.

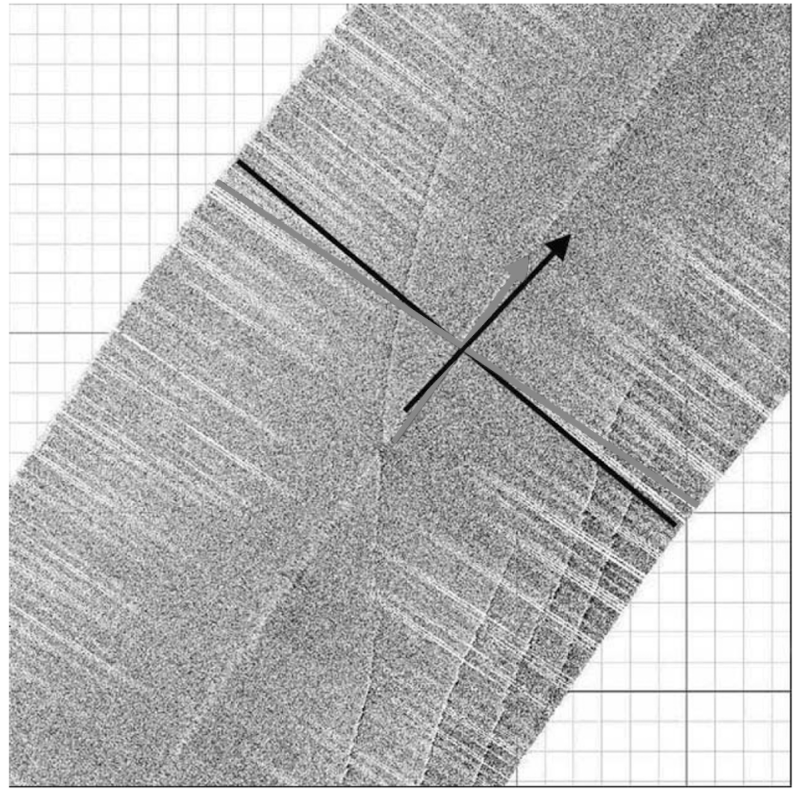

Fig. 7. An example of mosaic image with many gaps.

Moreover, we should keep in mind that the number of "pings" is not an independent variable proportional to the real space occupation. The surveying speed and footprint's width are also involved, which makes the criteria for reference window definition quite complicate. So far, we can only adjust the averaging window according to the sense of geology knowledge subjectively.

\section{PATCHING GAPS IN MOSAIC IMAGES}

The purpose of a sidescan sonar survey is to determine the geographical location of targets or seabed features. The sonar data therefore need to be assembled into a "mosaic" which represents the geophysical features of the seabed. The data for each ping are projected as in a waterfall display, but the ping lines are placed on a map, perpendicular to the path of the towfish. Because this path is not always straight, the projected line segments are not all parallel to each other (unlike a waterfall display). The result can be seen in Fig. 7 gaps appear on the outer edge of the swath where it curves.

The gaps are certainly not a desirable feature in our image. Several processes have been suggested to deal with them, such as interpolation, or repetition of the surrounding data to fill the empty pixels $[6,8]$. These processes can successfully smooth the image. However, no theoretical justification has been produced for why the gaps should be patched.

One of the assumptions in the mosaicking process is that the sonar array scans only a "line segment". However, this assumption is untrue. The sonar signal has a finite width, and it spreads as it travels. Therefore the footprint of one ping is actually a fan-shaped segment rather than a line, as shown in Fig. 8.

As Fig. 8 shows, the area of the swath furthest from the 


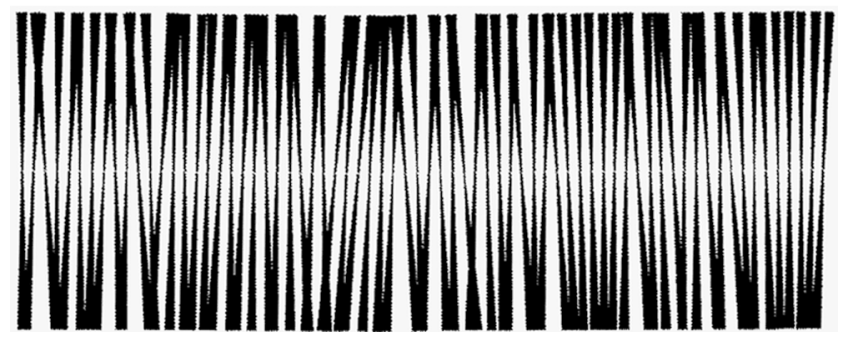

Fig. 8. Fan-shaped sonar footprints. Horizontal axis denotes the track of sonar moving; the vertical axis denotes the across track footprints.

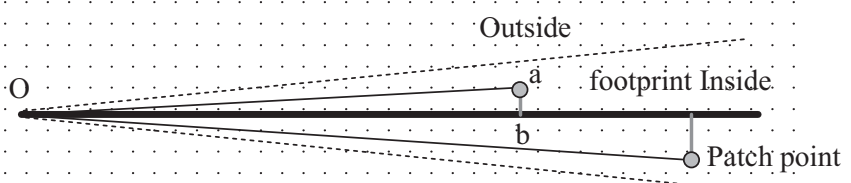

Fig. 9. Patching method based on the fan-shaped footprint.

towfish is actually scanned more than areas near the path of the towfish, because of the way the sonar beam spreads out. In a mosaic, therefore this area should not be blank. There should not be more gaps than in the middle of the swath. So in most cases, the gaps should be patched not only because they are bad for image continuity, but also because they are theoretically unjustified.

Figure 9 shows a patching method based on a fan-shaped sonar footprint. The mosaic image is first plotted in the traditional way, with all the data projected as line segments. After that, all empty pixels on the image are checked to find if they are within the footprint of any sonar ping. If they are not, no patching will be carried out. If they are, the nearest ping (defined by the perpendicular distance to the line segment) is selected as the data source. The empty pixels are assigned a raw intensity level from the data for that ping, based on their distance from the towfish $(\mathrm{O})$. In Fig. 9, the empty pixel " $\mathrm{a}$ " is assigned the same intensity as $b$. When this patching method is applied to Fig. 7, the result is as shown in Fig. 10. The continuity seems perfect.

When the pixel is actually covered by more than one fanshaped footprint, it seems reasonable that we can introduce some weighting algorithm to combine the contributions of all pings into the pixel's intensity. However, it usually causes some smoothing effect, which makes the patched lines themselves become false features. An example of similar situation is shown in Fig. 11. The main reason for this is the background intensities of sidescan images are generally with high frequency noises. Therefore choose only one raw datum for each pixel might be a better solution.

\section{CONCLUSION}

This paper describes three important processing procedures

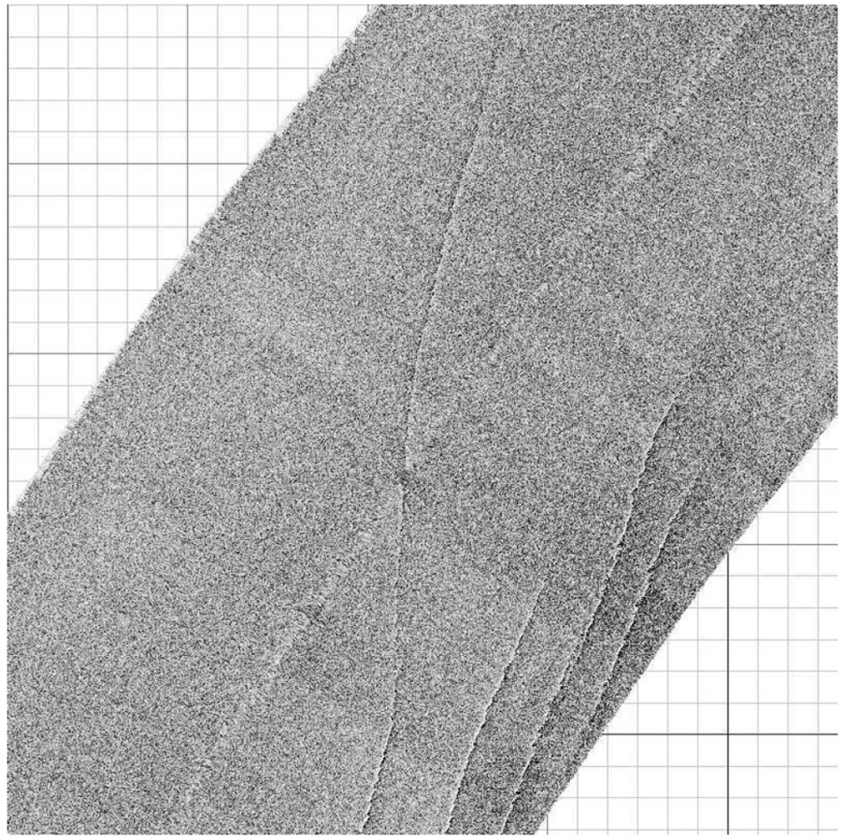

Fig. 10. Mosaic image patched using the footprint process.

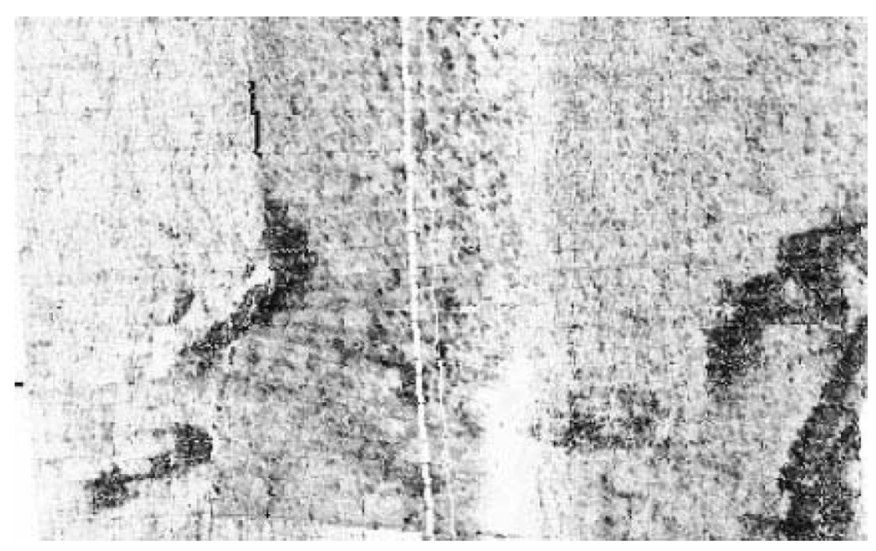

Fig. 11. Mosaic image patched by interpolation algorithm [6]. The most across-track gray lines overlay the images are patched lines.

for sidescan sonar data. Two of the processes address the uneven brightness inherent in the sonar data. One is to patch the gaps in mosaic images. The three processes are listed below:

1. Processing to compensate the beam pattern (angle and intensity) varies across the swath.

2. Normalizing ping energy levels to remove the effects of sudden energy level changes, caused largely by towfish changes in attitude.

3. Patching gaps using a method supported theoretically by the shape of the sonar footprint.

All of these processes are successfully applied in our current projects. 


\section{REFERENCES}

1. Blondel, Ph., "Automatic mine detection by textural analysis of COTS sidescan sonar imagery," International Journal of Remote Sensing, Vol. 21, No. 16, pp. 3115-3128 (2000).

2. Brown, C. J., Cooper, K. M., Meadows, W. J., Limpenny, D. S., and Rees, H. L., "Small-scale mapping of sea-bed assemblages in the Eastern English channel using sidescan sonar and remote sampling techniques," Estuarine, Coastal and Shelf Science, Vol. 54, pp. 263-278 (2002).

3. Charlot, D., Girault, R., and Zerr, B., "DELPH-SONAR: A compact system for the acquisition and processing of side scan sonar images," OCEANS'94., Oceans Engineering for Today's Technology and Tomorrow's Preservation Proceedings, Vol. 2, pp. II/428-432 (1994).

4. Chavez, P. S., Jr., Isbrecht, J., Galanis, P., Gabel, G. L., Sides, S. C., Soltesz, D. L., Ross, S. L., and Velasco, M. G., "Processing, mosaicking and man- agement of the Monterey Bay digital sidescan-sonar images," Marine Geology, Vol. 181, pp. 305-315 (2002).

5. Fish, J. P. and Carr, H. A., Sound Underwater Images- A Guide to the Generation and Interpretation of Side Scan Sonar Data, American Underwater Search and Survey, Lower Cape Publishing, Orleans, MA, 188p (1990).

6. Hsueh, D. Y., Development of Sidescan Sonar Image Mosaicing Software, Master Thesis, Institute Of Applied Marine Physics and Undersea Technology, National Sun Yat-sen University, Kaohsiung, Taiwan (2007).

7. Huvenne, V. A. I., Blondel, Ph., and Henriet, J.-P., "Textural analyses of sidescan sonar imagery from two mound provinces in the Porcupine seabight," Marine Geology., Vol. 189, pp. 323-341 (2002).

8. Johnson, H. P. and Helferty, M., "The interpretation of side-scan sonar," Reviews of Geophysics, Vol. 28, No. 4, pp. 357-380 (1990). 